\title{
Consenso en el diagnóstico y tratamiento de la diabetes tipo 1 del niño y del adolescente
}

\author{
SYLVIA ASENJO ${ }^{1,2}$, SANTIAGO MUZZO B. ${ }^{1,3}$, MARÍA VIRGINIA PÉREZ ${ }^{1,4}$, \\ FRANCISCA UGARTE P. ${ }^{1,5}$, MARÍA EUGENIA WILLSHAW ${ }^{1,6}$ \\ Rama de Endocrinología, Sociedad Chilena de Pediatría
}

\section{Prólogo}

La Rama de Endocrinología de la Sociedad Chilena de Pediatría, elaboró este documento de consenso para el manejo de niños y adolescentes con diabetes tipo 1 (DM1).

Por su parte, el Ministerio de Salud incorporó la DM1 dentro del Plan Auge en Abril de 2004 y generó una normativa técnica, la cual fue considerada en este consenso.

Los contenidos del consenso incluyen: criterios diagnósticos y clasificación actual, objetivos del tratamiento, nuevas insulinas y esquemas insulínicos, manejo de situaciones especiales, pesquisa de complicaciones agudas y crónicas, y alimentación en pacientes con diabetes tipo 1 .

\section{Introducción}

La Diabetes Mellitus (DM) es una enfermedad crónica que tiene diferentes etiologías y se caracteriza por hiperglicemia, resultado de un déficit en la secreción de la insulina de su acción o de ambas.
La diabetes tipo 1 se caracteriza por destrucción paulatina de las células ${ }^{2}$ pancreáticas, por un proceso inmuno mediado en un huésped susceptible que ha sido expuesto a un gatillante ambiental, lo que conduce a un déficit absoluto de insulina. En $90 \%$ de los pacientes se detectan anticuerpos anti-islotes(ICA), anti-decarboxilasa del ácido glutámico (antiGAD), anti-insulina (IAA) y transmembrana tirosin-fosfatasa (IA-2); $10 \%$ de los casos son considerados idiopáticos y no presentan marcadores serológicos de autoinmunidad.

Los síntomas clásicos se presentan cuando se ha perdido cerca del $90 \%$ de la capacidad funcional de las células $\beta$. La incidencia varía de una zona geográfica a otra y en diferentes grupos étnicos de un mismo país. En Chile, se estima que en los menores de 15 años, la incidencia es de 6,58 por 100000 .

La DM1 es poco frecuente bajo el año de edad, presenta mayor prevalencia entre $\operatorname{los} 4 \mathrm{y}$ 6 años y aún mayor entre los 10 y 14 años. Actualmente, se observa una tendencia al aumento en la incidencia de DM 1, incluso en niños menores de 5 años.

1. Médico Endocrinólogo Pediátrico.

2. Servicio de Pediatría, Departamento de Pediatría, Facultad de Medicina Universidad de Concepción.

3. Instituto de Nutrición y Tecnología de los Alimentos (INTA), Universidad de Chile.

4. Servicio de Pediatría, Hospital San Juan de Dios.

5. Servicio de Pediatría, Hospital Exequiel González Cortés.

6. Servicio de Pediatría, Hospital Militar. 


\section{Criterios diagnósticos y clasificación de la Diabetes Mellitus}

Los pacientes pueden presentar un debut clásico con las siguientes manifestaciones clínicas: poliuria, polidipsia, polifagia y baja de peso; que orientan el diagnóstico, el que es formulado en un período variable de 2 a 6 semanas; en algunos casos existe una rápida progresión de los síntomas, los que se acompañan de vómitos, deshidratación, y grados variables de compromiso de conciencia, cuadro correspondiente a cetoacidosis diabética.

La ADA (Asociación Americana de Diabetes) definió en 2004 los siguientes criterios diagnósticos para Diabetes Mellitus:

a) Síntomas clásicos de diabetes y una glicemia casual (a cualquier hora del día) igual o mayor a $200 \mathrm{mg} / \mathrm{dL}$.

b) Dos glicemias en ayunas (por lo menos de ocho horas) igual o mayor a $126 \mathrm{mg} / \mathrm{dL}$.

c) Glicemia igual o mayor a $200 \mathrm{mg} / \mathrm{dL}$ dos horas después de una carga de glucosa durante una prueba de tolerancia a la glucosa oral (tabla 1). Define intolerancia a la glucosa una glicemia entre 140 y $199 \mathrm{mg} / \mathrm{dL}$ a las 2 horas de la prueba de tolerancia oral a la glucosa y glicemia de ayuno alterada a una glicemia de ayuno entre 100 y $125 \mathrm{mg} / \mathrm{dL}$. A estos dos criterios se les ha denominado últimamente pre-diabetes indicando mayor riesgo de desarrollar diabetes.

La clasificación actualmente utilizada, es una clasificación etiológica y es la recomendada por la ADA en 1997 y por el Comité de Expertos para la Clasificación y Diagnóstico de la Diabetes (OMS, 1998). Clasifica la diabetes mellitus (DM) en:

a) DM tipo 1 (inmunomediada (1a) e idiopática (1b)). b) DM tipo 2 la que puede variar desde predominantemente insulina-resistente con deficiencia relativa de insulina a un defecto preferentemente secretor con o sin resistencia insulínica.

c) Tipos específicos de DM que comprende: Defectos genéticos de la función de la célula B, MODY (Maturity Onset Diabetes of the Young), de la acción de la insulina, enfermedades del páncreas exocrino, endocrinopatías, acción de drogas o tóxicos, infecciones y en otros casos asociada a síndromes genéticos.

d) DM gestacional es aquella que se presenta en el curso del embarazo.

En ocasiones la diabetes mellitus puede ser de inicio insidioso y presentar algunas dificultades en su clasificación. Para diferenciar la DM1 de la DM2, especialmente en adolescentes obesos, es importante considerar el antecedente familiar diabetes y la presencia de signos de resistencia insulínica como acantosis nigricans, frecuentes en DM2. Exámenes que ayudan al diagnóstico diferencial son la determinación de anticuerpos anti-islote (ICA), anti GAD, antiinsulina y niveles de péptido $\mathrm{C}$. La concentración de insulina sirve sólo si es medida antes del inicio del tratamiento insulínico.

\section{Objetivos del Tratamiento}

Los objetivos del tratamiento son lograr un buen control metabólico, crecimiento y desarrollo normal, evitar las complicaciones agudas y prevenir las crónicas. Asimismo, lograr un efectivo automonitoreo y autocuidado de la enfermedad, estimulando una autonomía progresiva y un equilibrio emocional adecuado.

El DCCT (Diabetes Control and Complications Trial) evidenció que el manejo intensificado

Tabla 1. Diagnóstico de laboratorio en diabetes mellitus

\begin{tabular}{llll} 
& Normal & Alteraciones & Diabetes \\
\hline Glicemia en ayunas & $<100 \mathrm{mg} / \mathrm{dL}$ & $\begin{array}{l}\text { Igual o mayor a } 100 \text { y menor a } 126 \mathrm{mg} / \mathrm{dL} \\
\text { Glicemia de ayuno alterada }\end{array}$ & Igual o mayor a $126 \mathrm{mg} / \mathrm{dL}$ \\
Glicemia a las $2 \mathrm{hrs}^{*}$ & $<140 \mathrm{mg} / \mathrm{dL}$ & $\begin{array}{l}\text { Igual o mayor a } 140 \text { y menor a } 200 \mathrm{mg} / \mathrm{dL} \\
\text { Intolerancia a la glucosa }\end{array}$ & Igual o mayor a $200 \mathrm{mg} / \mathrm{dL}$ \\
\hline
\end{tabular}

TTGO : $1.75 \mathrm{~g}$ de glucosa por kilo de peso (máximo $75 \mathrm{~g}$ ). 
(múltiples dosis de insulina) de la DM1 reduce significativamente las complicaciones a largo plazo, especialmente las microvasculares; posteriormente el estudio EDIC demostró la prolongación del efecto beneficioso del tratamiento intensificado.

El valor de la hemoglobina glicosilada (HbA1c) refleja la concentración de las glicemias de 12 semanas previas y representa el mejor parámetro de control metabólico. Este examen se debe efectuar cada tres meses. La ADA propone valores de glicemias y HbA1c según la edad (tabla 2), considerando las deletéreas consecuencias de la hipoglicemia a menor edad del paciente.

El tratamiento integral del diabético contempla como pilares fundamentales: terapia insulínica, plan de alimentación según el esquema insulínico utilizado, automonitoreo y educación continua. El Protocolo AUGE DM tipo 1 garantiza la atención por equipo multidisciplinario incluyendo médico pediatra o internista especialista o con capacitación en diabetes, nutricionista, enfermera y psicólogo (deseable); los insumos para el tratamiento y automonitoreo, así cómo los exámenes necesarios para evaluación de control metabólico y detección precoz de complicaciones.

\section{Tipos de insulina y esquemas terapéuticos}

Las insulinas empleadas hoy en día, son insulinas humanas de recombinación genética. En niños y adolescentes las más utilizadas y según sus tiempos de acción se clasifican en: insulinas de acción rápida, análogos de acción rápida (aspártica, lispro, glulisina), insulina de acción intermedia (NPH) y análogos basales de acción prolongada (glargina y detemir) (tabla 3). Los análogos de insulina corresponden a insulinas modificadas en algunos aminoácidos, que cambian sus características farmacodinámicas y sus tiempos de acción.

Los análogos de acción ultrarrápida tienen un inicio de acción más breve y menor tiempo de acción que la insulina regular. Pueden administrarse inmediatamente antes de las comidas, reduciendo las hiperglicemias post-prandiales y las hipoglicemias nocturnas. También se pueden usar por vía endovenosa, pero sin ventajas sobre la insulina regular en el empleo en esta vía.

Tabla 2. Glicemias y hemoglobinas glicosiladas recomendables

\begin{tabular}{|c|c|c|c|c|}
\hline \multirow[t]{2}{*}{ Edad } & \multicolumn{2}{|c|}{ Glicemias $\mathbf{m g} / \mathbf{d L}$} & \multirow[t]{2}{*}{ HbA1c \% } & \multirow[t]{2}{*}{ Fundamentos } \\
\hline & Preprandial & Nocturna & & \\
\hline$<6$ años & $100-180$ & $110-200$ & $<8,5->7,5$ & Alto riesgo hipoglicemia \\
\hline 6-12 años & $90-180$ & $100-180$ & $<8$ & Riesgo de hipoglicemia \\
\hline 13-19 años & $90-130$ & $90-150$ & $<7,5$ & \\
\hline
\end{tabular}

Diabetes Care 2005; 28: 186-212

Tabla 3. Tipos de insulina y sus tiempos de acción

\begin{tabular}{llllc}
\hline Insulina & Tipo de Insulina & $\begin{array}{c}\text { Inicio de } \\
\text { acción }\end{array}$ & $\begin{array}{c}\text { Máxima } \\
\text { acción }\end{array}$ & $\begin{array}{c}\text { Duración de } \\
\text { acción }\end{array}$ \\
\hline NPH & Regular & $2 \mathrm{hr}$ & 6 horas & $12 \mathrm{hr}$ \\
Glargina (Lantus) & Análogo de acción basal & $2 \mathrm{a} 4 \mathrm{hr}$ & Sin peak & $24 \mathrm{hr}$ \\
Detemir (Levemir) & Análogo de acción basal & $15 \mathrm{a} 30 \mathrm{~min}$ & Sin peak & $12 \mathrm{hr}$ \\
Cristalina & Rápida & $30 \mathrm{~min}$ & $3 \mathrm{hr}$ & $6 \mathrm{hr}$ \\
Lispro (Humalog) & Análogo de acción ultrarrápida & $<15 \mathrm{~min}$ & $30 \mathrm{a} 90 \mathrm{~min}$ & $4 \mathrm{hr}$ \\
Aspartica (Novorapid) & Análogo de acción ultrarrápida & $<15 \mathrm{~min}$ & $30 \mathrm{a} 90 \mathrm{~min}$ & $4 \mathrm{hr}$ \\
Glulisina (Apidra) & Análogo de acción ultrarrápida & 10 a $15 \mathrm{~min}$ & $30 \mathrm{a} 60 \mathrm{~min}$ & $4 \mathrm{hr}$ \\
\hline
\end{tabular}


La insulina isofana NPH es la más usada como insulina de acción intermedia, tiene la ventaja que puede ser mezclada en la misma jeringa sin experimentar ninguna interacción con insulina regular y análogos de acción ultrarrapida.

Los análogos de insulinas basales (glargina y detemir) permiten un efecto insulínico más predecible comparado con la NPH; su uso está formalmente aprobado en mayores de 6 años, pero existe información en la literatura de empleo exitoso en edades menores. El tiempo de acción de la insulina glargina es de 24 horas pero en algunos casos se observa que no alcanza este período, por lo que debe administrarse una segunda dosis.

La insulina detemir debe administrarse en 2 a 3 dosis diarias.

Los distintos esquemas de insulina buscan imitar la secreción normal de insulina por el páncreas, que contempla una secreción basal baja y bolos de secreción en relación a la ingesta de alimentos. Por esto todos los esquemas contemplan insulinas basales y prandiales. Las insulinas basales más utilizadas en niños y adolescentes, son la insulina intermedia NPH (en 2 ó 3 dosis) y la insulina glargina (análogo de acción prolongada, en dosis única o 2 dosis). Las insulinas prandiales de acción rápida más utilizadas son la insulina regular y análogos de acción ultrarrápida. Las insulinas premezcladas, en general no se usan en niños y adolescentes ya que no permiten la flexibilidad en las dosis según glicemia, aporte de hidratos de carbono y ejercicio a realizar.

El esquema a utilizar depende por una parte de la disponibilidad de las distintas insulinas así como del régimen de vida del paciente: horarios de estudio, hábitos de alimentación y de actividad física. El protocolo AUGE DM tipo 1 incluye insulina NPH, cristalina y ultrarrápida para menores de 20 años. La insulina Glargina no está contemplada actualmente, a pesar de que la mayoría de las Isapres la han incorporado.

En escolares chilenos uno de los esquemas más utilizados es insulina NPH como basal en 2 ó 3 dosis e insulina cristalina o ultrarrápida como insulina prandial; en niños y jóvenes con horarios irregulares de alimentación insulina glargina con insulina prandial cristalina $\mathrm{y} / \mathrm{o}$ ultrarrápida, constituye uno de los esquemas de elección. Sin embargo, múltiples esquemas pueden permitir un buen control metabólico y debe seleccionarse el que aporte o condicione una mejor calidad de vida, considerando los hábitos familiares y las preferencias de los padres y/o del paciente.

Existe acuerdo en que los esquemas intensificados, con múltiples dosis de insulina y controles glicémicos en el día, son los que se asocian con un mejor control metabólico y previenen las complicaciones crónicas.

El ajuste de dosis de insulinas basales se realiza considerando las glicemias preprandiales y el horario de máxima acción de la insulina; en cambio las dosis de insulina prandial se ajustan considerando la variación de la glicemia pre vs post prandial, la ingesta de hidratos de carbono de esa comida y el ejercicio realizado o por realizar en el día.

En general, la dosis total diaria de insulina utilizada por los pacientes varía entre 0,75 y 1 $\mathrm{U} / \mathrm{kg}$ por día, de la cual cerca del $50 \%$ es insulina basal. En adolescentes, por presentar resistencia fisiológica a insulina, es frecuente que requieran dosis totales más altas.

Recientemente han llegado a Chile las bombas de infusión continua, ampliamente utilizadas en algunos países desarrollados. Estos dispositivos entregan insulina ultrarrápida subcutánea en forma continua, de acuerdo a un programa que considera distintas dosis basales durante el día (habitualmente de 1 a 3 diferentes dosis) y bolos de insulina prandiales. Se utilizan en pacientes con buena adherencia al tratamiento y la mayoría de los autores señalan que mejoran la calidad de vida de los pacientes. Por el mayor costo tanto del dispositivo mismo como de los insumos requeridos para su utilización, por ahora no están consideradas dentro de los programas AUGE DM ministeriales ni de Isapres.

\section{Insulinoterapia en situaciones especiales}

a) Días de enfermedad:

Durante los días de enfermedad, así como frente a situaciones de estrés y transgresiones alimentarias, los pacientes suelen presentar hiperglicemias sin cetonemia. En estos casos se aconseja mantener la insulina basal y au- 
mentar la insulina prandial en $10 \%$. En caso de hiperglicemias postprandiales sobre $250 \mathrm{mg} / \mathrm{dL}$ puede administrar refuerzos de insulina ultrarrápida $(0,05 \mathrm{U} / \mathrm{Kg}$ o $50 \%$ de la dosis de refuerzo preprandial para esa misma glicemia). Debe controlarse la glicemia capilar frecuentemente y medir cetonuria o cetonemia si las glicemias persisten elevadas. Debe prevenirse la deshidratación, aumentando la ingesta de líquidos.

Ocasionalmente, frente a cuadros digestivos con vómitos y/o diarrea, o bien por rechazo alimentario, puede producirse una hipoglicemia. En estos casos se aconseja disminuir la insulina basal y la prandial en 10 a $15 \%$ de acuerdo a la glicemia y administrar colaciones de 10 a $15 \mathrm{~g}$ de hidratos de carbono si se produce hipoglicemia (tabla 4).

Debe consultar en servicio de urgencia cuando el diagnóstico de la enfermedad no está claro, los vómitos son persistentes, la glicemia continúa subiendo a pesar de dosis ajustadas de insulina y existe cetonuria elevada y persistente, así como cuando existe hipoglicemia, decaimiento importante, compromiso de conciencia, deshidratación o dolor abdominal intenso.

b) Paciente con hiperglicemia y cetonemia positiva leve, sin signos clínicos de acidosis ni deshidratación. En este caso se recomiendan las mismas medidas que en a), pero puede ser necesario aumentar hasta en $15 \%$ las dosis totales de insulina para lograr controlar las hiperglicemias.

c) Paciente con hiperglicemia, cetonemia moderada y sin acidosis, cambiar a esquema de insulina cristalina cada 4 a 6 h, ajustando según glicemia capilar antes y 2 ó 3 h después de cada dosis.

d) Cirugía programada en un paciente conocido: en estos casos se prefiere el día de la operación esquemas de insulina cristalina cada 4 a 6 horas, controlando la glicemia intraoperatoria en forma horaria, regulando el aporte de glucosa según glicemias y administrando insulina cristalina ev $(0,05$ a $0,1 \mathrm{U} / \mathrm{Kg})$ si la glicemia sobrepasa los $250 \mathrm{mg} / \mathrm{dL}$.

e) Cirugía de urgencia: cuando ya han recibido sus dosis de insulina basal y tendrán ayuno, se prefiere utilizar suero glucosado al 2,5 a $5 \%$, administrar insulina endovenosa en caso de glicemia sobre $250 \mathrm{mg} / \mathrm{dL}$ en dosis iniciales de $0,05 \mathrm{U} / \mathrm{Kg} / \mathrm{h}$ en goteo continuo y ajustar según las glicemias el aporte de glucosa y de insulina.

Algunos pacientes utilizan fórmulas de ajuste de dosis que consideran la sensibilidad a la insulina (disminución de la glicemia por unidad de insulina administrada) y la carga de hidratos de carbono a recibir. En los casos antes señalados debe aumentarse en 10 a $15 \%$ la dosis calculada y si existe cetonemias positivas aumentar hasta $20 \%$ la dosis calculada.

\section{Complicaciones agudas}

Las complicaciones agudas de la DM corresponden a la hipoglicemia y la cetoacidosis diabética.

La hipoglicemia, definida por distintos autores como glicemia menores de $50 \mathrm{mg} \%$ o 60 $\mathrm{mg} \%$, se manifiesta por síntomas autonómicos y neuroglucopénicos, como palidez, temblor, sudoración fría, obnubilación, puede llegar a la pérdida de conocimiento, convulsiones y muerte. Requiere intervención rápida, administración de líquidos azucarados si el paciente está conciente y puede deglutir. Idealmente estos líquidos deben ser sin proteínas ni grasas, para una absorción más rápida. $\mathrm{Si}$ el paciente no está conciente $\mathrm{y} / \mathrm{o}$ no puede deglutir, administrar glucagón I.M. (30 ug/kg con un máximo de

Tabla 4. Días enfermedad, laboratorio y conducta

\begin{tabular}{llccc}
\hline \multicolumn{4}{c}{ Glicemia $(\mathbf{m g} / \mathbf{d L})$} \\
\hline Cuerpos cetónicos & $<80$ & $80-250$ & $250-400$ & $>400$ \\
Negativo & $\begin{array}{l}\text { Mantener o disminuir 10\% insulina basal y } \\
\text { disminuir insulina prandial 10 a 15\% }\end{array}$ & Dosis habitual & $+10 \%$ DDT* & $+20 \%$ DDT \\
Positivo & Mantener insulina basal & Dosis habitual & $+20 \%$ DDT & $+20 \%$ DDT \\
\hline
\end{tabular}

*DDT: Dosis Diaria Total 
$1 \mathrm{mg} ; 1 \mathrm{amp}(1 \mathrm{mg}$ ) en mayores de 6 años y $1 / 2$ amp (0,5 mg) en menores de 6 años). Si el paciente está en un centro asistencial y dispone de vía venosa puede administrarse suero glucosado en bolo $(2 \mathrm{cc} / \mathrm{kg}$ de suero glucosado al 10\%) y luego mantención suero glucosado (3 a $5 \mathrm{mg} / \mathrm{kg} / \mathrm{min}$ ), para mantener una glicemia cercana a $100 \mathrm{mg} / \mathrm{dL}$.

La cetoacidosis diabética (CAD) se caracteriza por intensificación de los síntomas clásicos de la enfermedad, a lo que se agrega deshidratación, vómitos, con o sin compromiso de conciencia. Su diagnóstico se confirma con una glicemia mayor de $250 \mathrm{mg} / \mathrm{dL}$, un $\mathrm{pH}<7,3$, bicarbonato $<15 \mathrm{mEq} / \mathrm{Lt}$, cuerpos cetónicos positivos en sangre y orina. Su manejo requiere hospitalización en UCI o cuidados intermedios. El protocolo AUGE DM-1 entrega un flujograma del manejo de CAD en niños y adolescentes (figura 1). El edema cerebral es la complicación más grave en el tratamiento de la $\mathrm{CAD}$ y principal responsable de la mortalidad de este cuadro clínico, se presenta entre las 24 y 48 horas de evolución después de haber presentado una mejoría del estado general y sin asociarse a ningún parámetro al diagnóstico. Se manifiesta por cefalea, náuseas, vómitos y compromiso sensorial y su tratamiento consiste en administrar manitol 0,5 a $1 \mathrm{~g} / \mathrm{kg}$ por vía endovenosa en 15-20 minutos y restricción de otros fluidos administrados por vía endovenosa.

\section{Alimentación del paciente diabético}

El plan de alimentación tiene como objetivo mantener un adecuado estado nutricional, permitir un desarrollo y crecimiento normal en el niño. La alimentación del niño y adolescente diabético debe ajustarse en base al aporte calórico según la edad, al igual que en el niño normal (tabla 6). Con un aporte calórico de 50 a $60 \%$ en base de hidratos de carbono, $20 \%$ de proteínas y $20 \%$ a $30 \%$ de grasas (ácidos grasos poliinsaturados, monosaturados y saturados 1:1:1), y colesterol < $300 \mathrm{mg} /$ día. En el cálculo

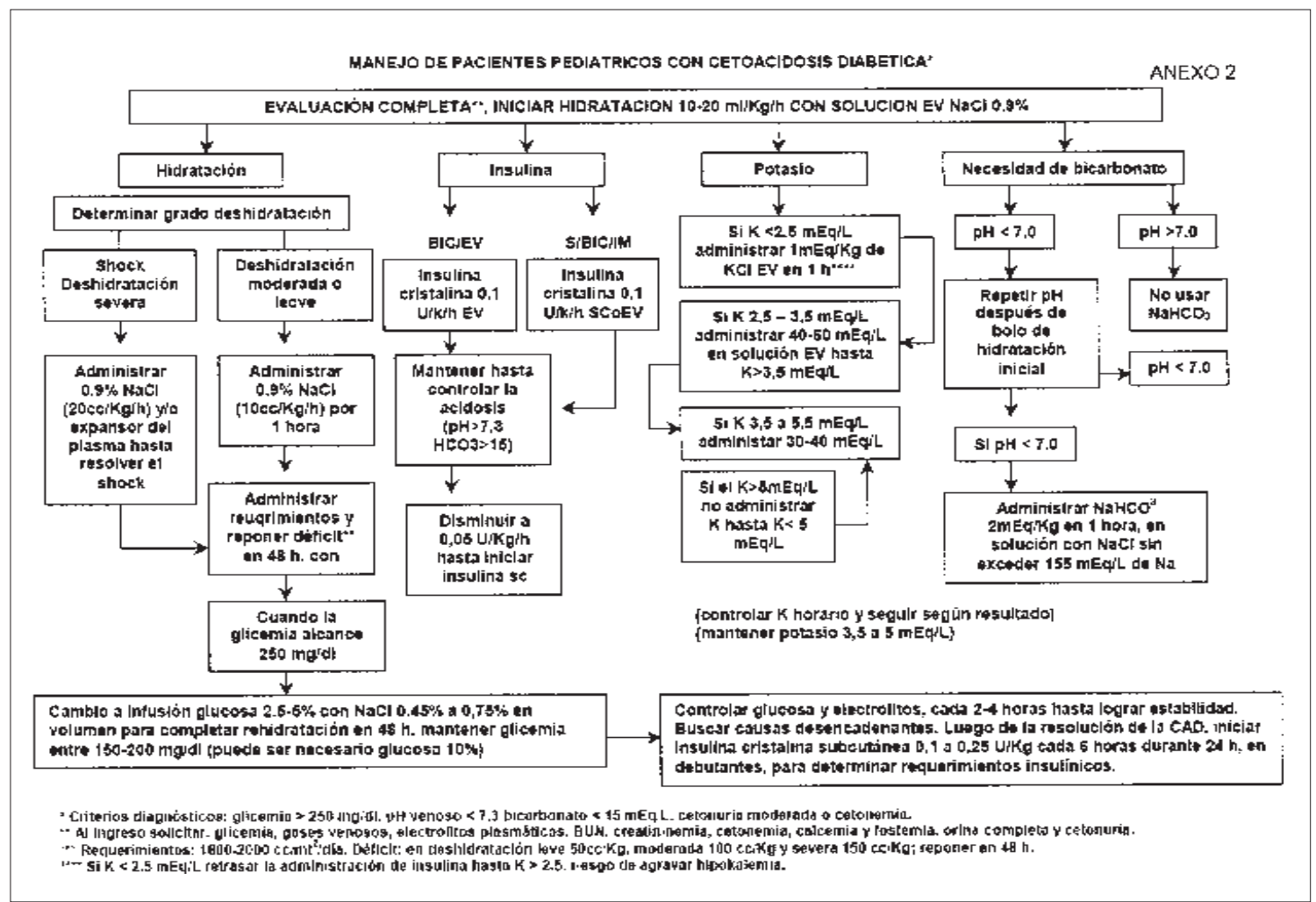

Figura 1. Tratamiento de la cetoacidosis diabética en el niño. Protocolo auge diabetes tipo 1. 
Tabla 5. Alimentación del niño diabético

\begin{tabular}{cl}
\hline & Calorías diarias \\
\hline $0-12$ años & $\begin{array}{l}1000 \mathrm{cal} 1 \mathrm{er} \text {. año + } \\
100 \mathrm{cal} / \text { año }\end{array}$ \\
$12-15$ años & \\
Mujeres & $1500-2000 \mathrm{cal}+$ \\
& $100 \mathrm{cal} /$ año sobre 12 \\
Varones & $2000-2500 \mathrm{cal}+$ \\
& $200 \mathrm{cal} /$ año sobre 12 \\
& \\
Nutrientes (\% de las calorías) & $55-60 \%$ \\
Hidratos de carbono & $15-20 \%$ \\
Proteínas & $<30 \%$ * \\
Grasas &
\end{tabular}

*Acidos grasos poliinsaturados, monosaturados y saturados 1:1:1. Colesterol $<300 \mathrm{mg} /$ día

de las calorías totales a aportar debe considerarse el grado de actividad física y el estado nutricional (tablas 5 y 6). La distribución de los hidratos de carbono durante el día depende del esquema insulínico empleado y debe ser flexible y adaptado a cada caso en forma individual.

\section{Detección de complicaciones crónicas}

Las complicaciones crónicas incluyen la microvasculares: nefropatía, retinopatía y neuropatía diabética. La presencia de hipertensión arterial y dislipidemia confieren un mayor riesgo cardiovascular al paciente diabético, por lo que dentro del seguimiento debe considerarse su detección y manejo.

El Protocolo AUGE DM tipo 1 propone realizar determinaciones anuales de microalbuminuria y creatininemia en pacientes con 5 o más años de evolución de la enfermedad. El consenso de ISPAD recientemente publicado aconseja determinar microalbuminuria anualmente en mayores de 11 años con 2 años de evolución y desde los 9 años con 5 años de duración de la diabetes. Para la detección de retinopatía el protocolo AUGE indica realizar fondo de ojo anual por oftalmólogo a partir de 5 años de diabetes, ISPAD sugiere las mismas edades y tiempos de evolución antes mencionadas, realizando fotografía de retina u oftalmoscopía con pupila dilatada, aunque esta última tiene menor sensibilidad. En relación a la detec-
Tabla 6. Aporte calórico según actividad física y estado nutricional (kcal/kg peso aceptable/día)

\begin{tabular}{|c|c|c|c|}
\hline & Liviana & $\begin{array}{l}\text { ctividad física } \\
\text { Moderada }\end{array}$ & Intensa \\
\hline Obeso & $20-25$ & 30 & 35 \\
\hline Normal & 30 & 35 & 40 \\
\hline Enflaquecido & 35 & 40 & $45-50$ \\
\hline
\end{tabular}

ción precoz de neuropatía no existe evidencia que señale a que edad se debe iniciar la pesquisa y prima el criterio clínico. Concerniente a la prevención de enfermedad macrovascular existe coincidencia en la determinación del perfil lipídico a partir de los 12 años y control cada 5 años en caso de normalidad o anual frente a dislipidemia o antecedentes familiares de enfermedad cardiovascular. La presión arterial debe ser controlada anualmente.

Existen otras patologías autoinmunes que se presentan con mayor frecuencia en pacientes con DM tipo 1, como la tiroiditis linfocitaria crónica y la enfermedad celíaca; por lo que también es necesaria su pesquisa. La norma ministerial aconseja determinar anualmente T4 y TSH en niños.

Si bien las complicaciones crónicas son más frecuentes en adultos, algunos estudios muestran que un $18,5 \%$ de pacientes con DM menores de 15 años las presentan. Este y otros estudios hacen aconsejable la detección precoz de complicaciones crónicas en la edad pediátrica. El protocolo AUGE DM-1 propone una pauta de manejo para pacientes con microalbuminuria y con HTA.

El mejor conocimiento de la Diabetes tipo 1, la mayor disponibilidad de insumos para el autocontrol y la existencia de múltiples tipos de insulina, así como de productos alimenticios libres de azúcar; permiten hoy en día un mejor control metabólico y calidad de vida de los pacientes con Diabetes tipo 1. Esto determina mayores posibilidades de un crecimiento y desarrollo normal y a futuro evitar las complicaciones crónicas de esta enfermedad. Los esfuerzos realizados a nivel país para el mejor manejo de la Diabetes tipo 1, nos han posicionado en un lugar destacado dentro de América 
Latina, alcanzado muchos centros nacionales niveles de control de sus pacientes comparables a centros internacionales reconocidos.

La continua investigación e incorporación de nuevas tecnologías a nivel mundial en el manejo de pacientes con Diabetes tipo 1, como sensores de glicemia a tiempo real y bombas de infusión continua "inteligentes"; así como los avances en terapia de trasplante pancreático y de células beta, y terapias inmunológicas (células madres y otros), plantea un futuro más promisorio para estos pacientes y la necesidad de actualización permanente de las normas y programas de tratamiento y seguimiento de pacientes con Diabetes Mellitus tipo 1.

\section{Referencias}

1.- American Diabetes Association. Diagnosis and classification. Diabetes care 2005; 28: 37-42.

2.- Craig ME, Hattersley A, Donaghue K: ISPAD Clinical Practice Consensus Guidelines 2006-2007. Definition, epidemiology and classification. Pediatric Diabetes 2006; 7: 343-51.

3.- Hanas $R$, Donaghue $K$, Klingensmith $G$, Swift P: Editors of the ISPAD Clinical Practice Consensus
Guidelines 2006-2007, Ragnar Hanas MD, PhD (2006) ISPAD Clinical Practice Consensus Guidelines 20062007; Pediatric Diabetes 7: 341-2.

4.- Australian Paediatric Endocrine Group: Guidelines on the management of type 1 Diabetes in children and adolescents. 2004. Chapter 12.

5.- Chase P: Understanding Diabetes 10th edition 2002. Barbara Davis Center for Childhood Diabetes University of Colorado Health Sciences Center.

6.- Silverstein J, Klingensmith $G$, Copeland K, et al: Care of Children and Adolescents With Type 1 Diabetes: A statement of the American Diabetes Association. Diabetes Care. 2005; 28: 186-212.

7.- Protocolo AUGE: Diabetes Mellitus tipo 1. Gobierno de Chile. Ministerio de Salud. 2004.

8.- Carrasco E, Angel B, Codner E, García D, Ugarte F, Bruzzone ME, Pérez F: Incidencia de diabetes tipo 1 en Santiago de Chile: análisis por comunas de la Región Metropolitana en el período 2000-2004. Rev Méd Chile 2006; 134: 1258-64.

9.- Eyzaguirre F, Peláez JM, Sepúlveda C, et al: Diabetes Mellitus tipo 1 (DM1) en niños menores de 5 años: Características al debut vs otros grupos etarios en Chile. Rev Chil Pediatr 2006; 77: 375-81.

10.- Gallardo V, Ugarte F, Barrera A, et al: Pesquisa precoz de nefropatía diabética en niños y adolescentes portadores de diabetes mellitus tipo 1 Rev Chil Pediatr 2005; 76: 252-8.

11.- Eyzaguirre F, Codner E: Análogos de insulina: en búsqueda del reemplazo fisiológico. Rev Méd Chile 2006; 134: 239-50. 